\title{
COLLAPSE OF THE NEUTRON STAR INDUCED BY PHASE TRANSITIONS AND NEUTRINO EMISSION FROM SN1987A
}

Mariko TAKAHARA ${ }^{a}$ and Katsuhiko SATO ${ }^{b}$

${ }^{a}$ Depaftment of Physics, Tokyo Institute of Technology, Tokyo, Japan Department of Physics, University of Tokyo, Tokyo, Japan

The neutrino burst from SN1987A were detected by KAMIOKANDE and also IMB in February this year. The neutrino events detected by KAMIOKANDE were clustered into three bunches; event number 1-5, 7-9, 10-12. The first two bunches of the neutrino events can be understood by the standard scenario of supernova explosions.

However, it is very difficult to explain the last bunch of the neutrino events by the standard scenario, especially the total energy of the neutrinos and the radius of the neutrino sphere; it is necessary at least several times of $10^{52}$ ergs to explain the total energy of the last three events. In order to explain this energy, we must consider the non-standard mechanism of the neutrino emission.

As a possible mechanism we investigated the energy release produced by the phase transitions of the high density matter (transitions to the pion condensed state or to the quark matter); the newly born neutron star collapses due to the phase transitions between the second and the third bunch of the neutrino events and releases the energy necessary for the last three events.

As for the normal equation of state (EOS) of neutron stars we adopted the ones suggested by Bethe and Johnson of Model I (BJ) for the standard EOS and Cohen et al. (CLRC) for the very stiff EOS. To these EOS we consider the phase transitions of the high density matter with no latent heat. To represent the phase transition very generally, we treated the critical densities (the density at which phase transition starts, $\rho_{c 1}$, or terminates, $\rho_{c 2}$ ) as the parameters. We assumed that the pressure is constant at $\rho_{\mathrm{c} 1}<\rho<\rho_{\mathrm{c} 2}$ and that the sound velocity equals to the light velocity at $\rho>p_{\mathrm{c}}$.

We consider the neutron star of $1.4 \mathrm{M}_{\odot}$ with normal EOS and set the central density equal to $\rho_{\mathrm{c} 1}$. Then $\rho_{\mathrm{c} 1}$ depends on the normal EOS and, as the stiffness of the normal EOS increases, $\rho_{\mathrm{c}} 1$ decreases; $\rho_{\mathrm{c} 1}$ $=9.8 \times 10^{14} \mathrm{gcm}^{-3}$ for BJ and $5.6 \times 10^{14} \mathrm{gcm}^{-3}$ for CLRC. Among the various choice of $P_{\mathrm{C} 2}$, we search the EOS which satisfy the following conditions; 1) the critical mass of the neutron star calculated in use of the EOS, $M_{c r}$, is larger than the observed mass of the neutron star 
1.4 $M_{\odot}$ 2) the released energy by the collapse of the neutron stars induced by phase transitions, $\Delta E$, is larger than several times of $10^{52}$ ergs necessary for last three events detected by KAMIOKANDE, 3 ) the EOS must be stiff enough so that the collapse of neutron star can be halted if a neutron star is left after this supernova explosion. The last condition is checked by the hydrodynamical calculation of the collapse of the neutron star.

In $F i g .1$ we plotted $M_{c r}$ and $\Delta E$ versus $P_{c 2}$. As phase transition becomes stronger, i.e., $P_{\mathrm{c} 2}$ becomes larger, $\mathrm{M}_{\mathrm{cr}}$ becomes smaller and $\Delta E$ becomes larger. However, if phase transitions are too strong, the collapse of the neutron star cannot be halted. For example, for the normal EOS of $B J$, the region of $\rho_{\mathrm{c} 2}>1.8 \times 10^{15} \mathrm{gcm}^{-3}$ is excluded by this condition. Therefore, very strong phase transitions are avoided by the conditions 1 ) and 3 ), while very weak transitions are avoided by the condition 2). Consequently moderately strong phase transitions are adequate for our purpose.

However, as is seen in Fig. 1, the absolute value of $\Delta \mathrm{E}$ depends sensitively on the normal EOS; the stiffer EOS can release larger energy. If we adopt $B J$, the releases energy is too small to explain the last three events. However, the enough energy is released for the very stiff EOS of CLRC. Therefore, we conclude that, if the normal EOS is sufficiently stiff, $\Delta \mathrm{E}$ is large enough to explain the last three events detected by KAMIOKANDE by this mechanism.

Fig. 1. The critical mass of neutron stars $M_{c r}$ (dashed lines) and the released energy by the collapse of the neutron star induced by phase transition $\Delta \mathrm{E}$ (solid lines) versus the strength of phase transition $\left(\rho_{\mathrm{c} 2}\right)$. As for the normal EOS, two normal EOS is considered; EOS of Bethe and Johnson (BJ) for standard EOS and Cohen et al. (CLRC) for very stiff EOS.

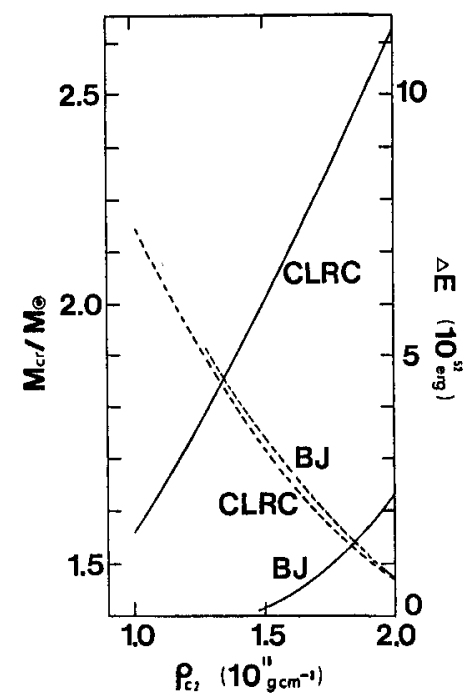

